\title{
Rutas gastronómicas como factor de desarrollo: Caso Esmeraldas-Ecuador
}

\section{Gastronomic routes as a factor of development: Case Esmeraldas-Ecuador}

\author{
MSc, César Gardel Villagómez Buele ${ }^{1}$ \\ cesar.villagomezb@uq.edu.ec \\ MSc, Marina Urbecí Arteaga Peñafiel ${ }^{2}$ \\ marina.arteagap@ug.edu.ec \\ MSc, Grace Helga Molina Bravo ${ }^{3}$ \\ grace.molinab@ug.edu.ec
}

Recibido 1/09/2018; Aceptado 1/11/2018 2018

\begin{abstract}
RESUMEN
Las rutas gastronómicas permiten desarrollar economías locales y posicionar destinos turísticos. La gastronomía de Esmeraldas es considerada la más afrodisiaca del Ecuador, sus recursos turísticos naturales y culturales son muy visitados por turistas; lo cual ha motivado al desarrollo del presente estudio considerando que en Ecuador aun no existen Rutas Gastronómicas. El objetivo de la investigación es diagnosticar el turismo gastronómico en los cantones Esmeraldas, Atacames y Rio Verde mediante una caracterización del destino y la aceptabilidad de la creación de una ruta gastronómica dentro de la Provincia de Esmeraldas, realizada in situ. Los resultados demuestran que la Provincia de Esmeraldas cuenta con potencial turístico gracias a sus playas y gastronomía mientras que la infraestructura turística, viabilidad, medios de transporte, calidad del servicio de alojamiento imperiosamente deben mejorarse a través de inversión pública y privada. Finalmente se obtuvo un grado de satisfacción alto en cuanto a la creación de una ruta gastronómica.
\end{abstract}

Palabras Clave: ruta gastronómica, desarrollo local, posicionamiento, turismo gastronómico, potencial turístico

\footnotetext{
${ }^{1}$ Universidad de Guayaquil, Guayaquil, Ecuador.

2 Universidad de Guayaquil, Guayaquil, Ecuador.

${ }^{3}$ Universidad de Guayaquil, Guayaquil, Ecuador.
}

Revista científica Ciencia y Tecnología Vol. 19 No 21 págs. 36-44 http://cienciaytecnologia.uteg.edu.ec 


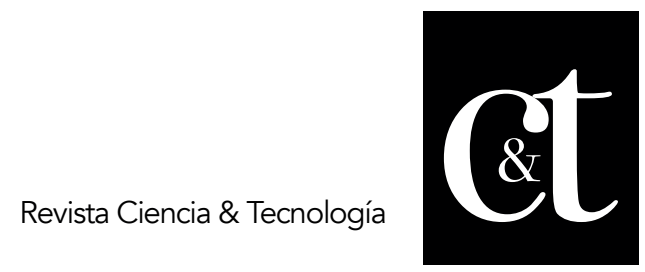

No. 21, 31 de enero de 2019

ISSN impreso: 1390 - 6321

\begin{abstract}
The gastronomic routes allow the development of local economies and the positioning of tourist destinations. The Esmeraldas's gastronomy is considered the most aphrodisiac of Ecuador, its natural and cultural tourist resources are very visited by tourists; which has motivated the development of the present study considering that in Ecuador there are still no Gastronomic Routes. The objective of the research is to diagnose gastronomic tourism in the cantons of Esmeraldas, Atacames and Rio Verde through a characterization of the destination and the acceptability of the creation of a gastronomic route within the Esmeraldas Province, carried out in situ. The results show that the Province of Esmeraldas has tourism potential thanks to its beaches and gastronomy, the tourist infrastructure, viability, means of transport, quality of the accommodation service must be improved through public and private investment. Finally, a high degree of satisfaction was obtained regarding the creation of a gastronomic route.
\end{abstract}

Keywords: gastronomic route, local development, positioning, gastronomic tourism, tourist potential

\title{
Introducción
}

La gastronomía es actualmente uno de los elementos clave para el desarrollo y la potenciación de un destino turístico (López-Guzmán y Jesús, 2011). Para Vázquez, Amador \& Arjona (2016) el turismo gastronómico puede desempeñar un papel relevante en la transmisión de valores tradicionales, la autenticidad y la identidad de un territorio. Además, en lugar de los preparativos de alimentos en restaurantes y hoteles para turistas, los turistas que viajan en busca de tener experiencia en alimentos y bebidas locales están dentro del ámbito del turismo gastronómico (Akgol 2012). En lo que respecta a turismo y alimentación, es un factor estrechamente asociado al turismo rural y, como tal, estrechamente ligado al desarrollo local por lo cual tampoco sorprende el surgimiento de un polo gastronómico fuera del ámbito urbano (Schlüter y Thiel Ellul, 2008).

El turismo gastronómico incluye visitas a productores de alimentos, festivales gastronómicos, restaurantes y lugares especiales relacionados con algunos alimentos especiales junto con degustar un plato especial, observar sus procesos de producción y preparación o comer un plato especial de la mano de un chef famoso, así como ver cómo se está preparando un plato determinado (Hall, Mitchell y Sharples, 2003, Hall y Mitchell, 2005). Indico así mismo Yuncu (2010) que un turista puede querer visitar una zona para tener una nueva experiencia cultural, o simplemente quieren probar los platos locales y los diferentes gustos que pertenecen a esa área también. Esto contribuirá a la sostenibilidad de los recursos de las regiones y también a las instalaciones turísticas alternativas que se pueden estructurar en regiones donde la cultura de los alimentos está cobrando importancia.

El Ministerio de Turismo (2007) define una ruta turística como: Una herramienta de planificación y desarrollo que nos permite alinear atractivos turísticos, productos/actividades y servicios bajo una temática claramente establecida, en torno al recorrido de un punto $A$ hasta un punto $B$, que cuenta con una zona de influencia determinada. Según la Organización Mundial del Turismo, existe una tendencia creciente al posicionamiento de muchos destinos turísticos como destinos 


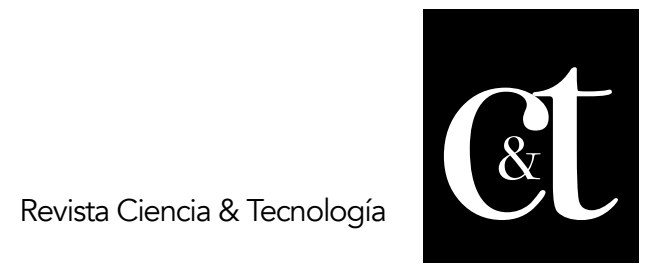

No. 21, 31 de enero de 2019

ISSN impreso: 1390 - 6321

gastronómicos (UNWTO, 2012). Para Buiatti (2011) tanto en países en vía de desarrollo, como en países industrializados, las rutas gastronómicas han sido creadas como plataformas para dinamizar económicamente zonas agrícolas que históricamente se quedaron al margen de las actividades turísticas, mediante una propuesta de turismo sostenible, apostando por la preservación del medioambiente y de las culturas locales.

El turismo culinario se define en la actualidad como una de las grandes apuestas para potenciar o consolidar destinos turísticos, debido a la importancia, cada vez mayor, que tiene para los viajeros el conocimiento de todo lo relacionado con la cultura gastronómica de los lugares que visita. (López-Guzmán, T., \& Jesús, M. M. 2011). En este sentido Folgado, Hernández y Campón (2011) consideran que el turismo es un sector en auge y con un gran margen de crecimiento, que debe basar su futuro en una planificación coordinada entre las entidades públicas y el sector privado. Mientras que Kresic y Prebezac (2011) sostienen que la utilización de recursos turísticos asociados a formas adecuadas de gestión, marketing e innovación puede convertirse en ventajas competitivas y proporcionar el desarrollo turístico sostenible a largo plazo.

\section{Desarrollo}

El binomio turismo-gastronomía está resultando un factor clave de áreas donde los recursos naturales, paisajísticos, culturales, arquitectónicos junto con la producción de alimentos diferenciados y de calidad resultan los únicos pilares o motores para su desarrollo económico. (Herrera y Arilla 2013). Para tal efecto Dos Santos y Antonini (2004) exteriorizan la valorización de la gastronomía típica de un lugar como producto turístico debe ser destacada por los guías como una herencia cultural resultante del desarrollo histórico, social, y de la creatividad del cocinero para crear nuevos sabores. Atendiendo a estas consideraciones Hernández (2011) sustenta que las rutas turísticas se ajusten a la organización comunitaria, contribuyendo a la vertebración territorial, evitando desequilibrios económicos socio-territoriales y promoviendo una visión holística o integral del patrimonio cultural basada en los usos y valores simbólicos autóctonos.

Partiendo de los supuestos anteriores Cristóvão, Tibério, Abreu (2008) afirman que los visitantes buscan los productos ligados a los sistemas productivos y alimentarios locales. De acuerdo con Vásquez, Morales-Fernández y Pérez (2012) las zonas rurales se deben asumir como verdaderos productos turísticos. Al mismo tiempo Bernard y Domínguez (2001) consideran que, ayudando a preservar las antiguas tradiciones y modos de vida expresados a través de los alimentos y de su preparación, y promoviendo el uso de ingredientes locales, se fomenta un tipo de turismo que sin lugar a duda es sustentable.

La gastronomía puede ser un componente sustancial para que un turista elija un destino. Con referencia Kozak (1997) declaro a la cultura, como elemento del turismo gastronómico, generalmente se expresa con la voluntad interna de las personas para conocer diferentes culturas. A este respecto, Akgol (2012) afirmó que el turismo gastronómico es de interés turístico, a través del aprendizaje de las características culturales de las zonas de acogida gracias a los platos locales de la zona, los turistas 


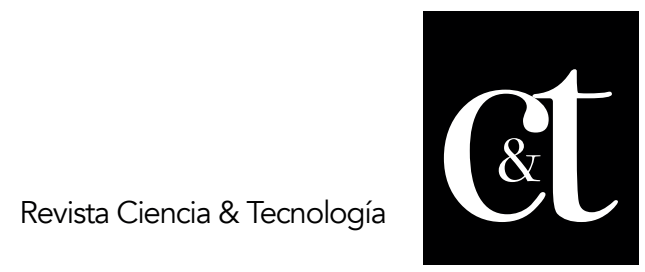

No. 21, 31 de enero de 2019

ISSN impreso: 1390 - 6321

gastronómicos también son turistas culturales al mismo tiempo. De igual manera para Cagli (2012) el turismo afecta a las personas y las naciones al establecer relaciones entre sí y también integra los fenómenos de la cultura, el patrimonio, la economía y el medio ambiente; por lo tanto, orientar su desarrollo, su coordinación y control son cuestiones sumamente cruciales.

La creación de productos como rutas turísticas, festivales gastronómicos, ferias, entre otras actividades puede ser dinamizada en el contexto de esa relación histórica presentada a partir de la alimentación (Martins, Baptista y Rocha, 2011). En efecto Ascanio (2009) manifiesta que el valor psicológico de estas rutas no está sólo en ir a un restaurante para degustar los platillos, se requiere algo más: se debe combinar esta experiencia gastronómica con una verdadera interpretación del contexto donde tuvo lugar la elaboración de estos manjares. Sin embargo, Vázquez, Amador y Arjona (2016) sostienen que el turista gastronómico no suele disponer de información ni de propuestas, claras y definidas, que le permitan valorar previamente todas las potencialidades de este tipo de rutas. Por esa razón Jeambey (2016) asevera que bien estructuradas y diseñadas, las rutas gastronómicas son importantes para el mantenimiento del paisaje natural, la valoración de la identidad local y el desarrollo económico del destino, siendo estos elementos clave para un desarrollo sostenible y un fuerte posicionamiento en el mercado turístico.

La gastronomía se vincula con el espacio en la producción agrícola en la transformación de los espacios naturales en paisajes que pueden ser utilizados por la actividad turística, desde la plantación de los alimentos hasta la transformación de los productos en platos tradicionales y típicos de cada región. (Mascarenhas y Gonçalves, 2010). No obstante, para Jeambey (2016) es de suma importancia que las rutas gastronómicas tengan una temática clara, una señalización, algún tipo de guía y la posibilidad de degustación y compra. Igualmente, importante es que los actores involucrados tengan claras sus responsabilidades y que trabajen colaborativamente.

Otro de los aspectos fundamentales es el servicio; al respecto da Silva Oliveira, do Amaral Moretti y Pozo (2015) complementan que los factores de hospitalidad, tales como la atención de sus expectativas, son identificables en todos los establecimientos como valores contributivos para el diseño y construcción de un diferencial de servicio. Otra forma de contribuir que considera Kim (1998) es que la diversidad de productos y servicios es primordial para el desarrollo superior del turismo de un determinado lugar. Es decir, un destino o atractivo exitoso necesita de una masa crítica de productos que posean viabilidad de la mercadología y llamamiento al consumidor. Como resultado Akgoz (2003) argumenta que el turismo es una fuente de ingresos para aquellos que brindan servicios a medida que las personas gastan para satisfacer sus necesidades cuando viajan.

Es importante realizar investigaciones referentes a rutas gastronómicas que permitan el desarrollo de destinos turísticos. En la actualidad aún no se realizan rutas gastronómicas en el Ecuador y tampoco existe propuestas para la provincia de Esmeraldas, por esa razón esta investigación se proyecta como objetivo el presentar resultados de un estudio de campo relacionado a la ciudad verde y su ruta 
gastronómica que atraviesa los cantones Río Verde, Esmeraldas y Atacames al norte del Ecuador, sur de Colombia; donde se puede disfrutar de parajes naturales únicos, a lo largo del perfil costanero, el clima cálido tropical y la gastronomía afrodisiaca a base de mariscos, pescado, verde y coco así como los bailes tradicionales y espacios para la práctica de deportes acuáticos permite desarrollar turismo de sol y playa, turismo gastronómico, ecoturismo, turismo de aventura y turismo cultural. En la Figura 1 se puede identificar la Ruta Gastronómica propuesta.

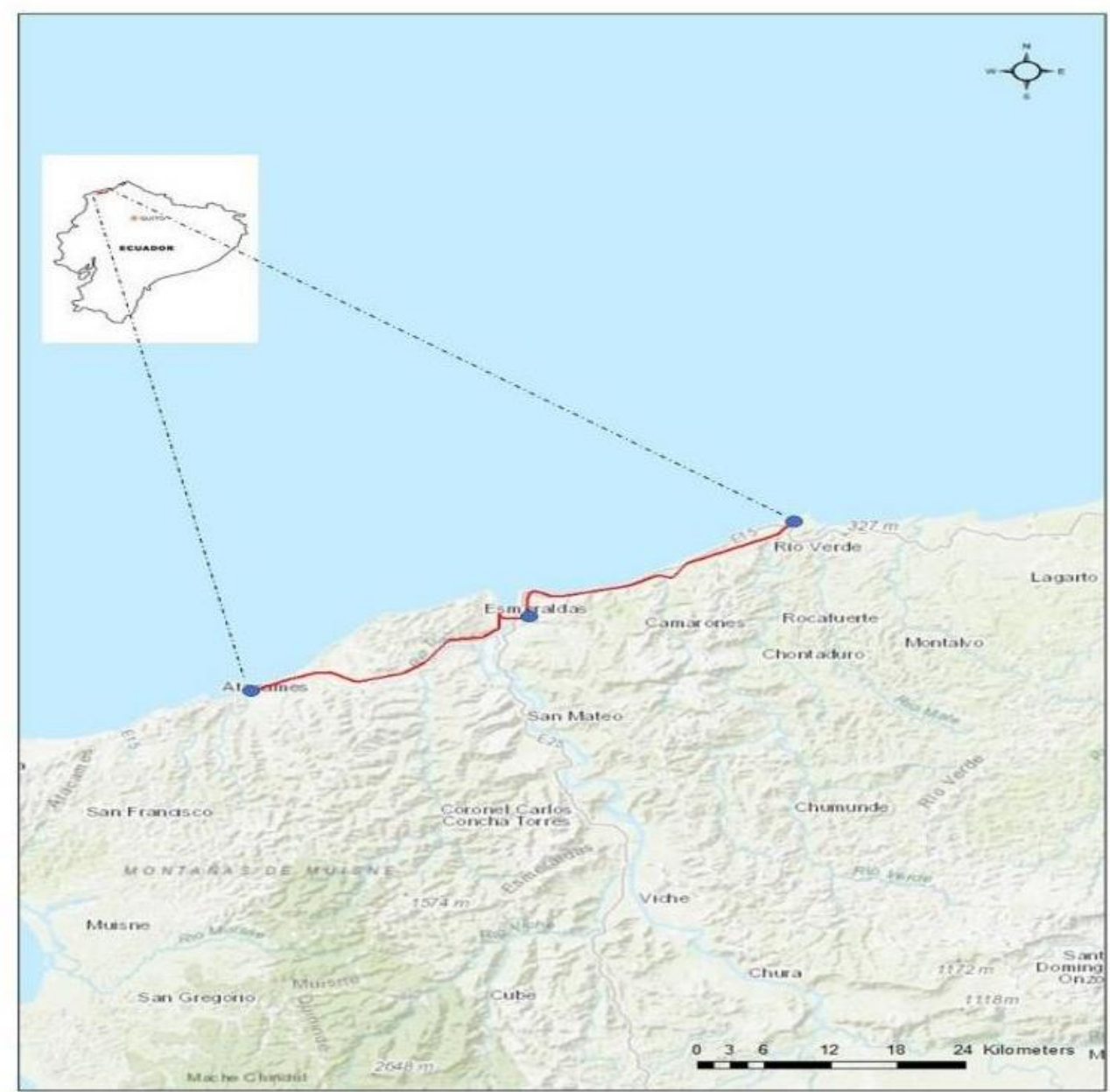

Gráfico 1. Ruta Gastronómica Atacames - Esmeraldas - Río Verde. Fuente: Elaboración Propia

\section{Método}

El método utilizado para el presente estudio empírico se basó en la realización de un trabajo de campo durante fines de semana entre los meses de Agosto y Septiembre del 2017 en los cantones Río Verde, Esmeraldas y Atacames de la provincia de Esmeraldas; donde se pudo recolectar la información mediante un cuestionario que contenía 14 preguntas, técnicamente organizado en dos secciones, para lo cual en la primera sección se requería de la información sociodemográfica de los visitantes y en la segunda sección se enfocaba en los requerimientos y mejoras de este importante destino turístico de Ecuador. La población objeto de estudio fueron visitantes mayores 


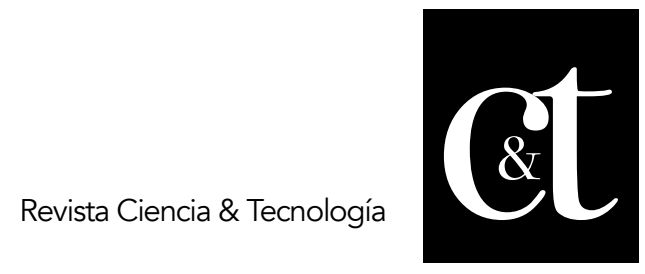

No. 21, 31 de enero de 2019

ISSN impreso: 1390 - 6321

de 18 años. Y se realizaron luego de haber degustado de los alimentos de estos sitios, para lo cual respondieron el cuestionario de forma independiente. Fueron empleadas una mezcla de técnicas de preguntas que conlleven a obtener resultados lo suficientemente confiables y se utilizaron preguntas estandarizadas con opciones múltiples y preguntas cerradas.

Se usaron 384 cuestionarios, siendo éste el tamaño de la muestra, para el presente estudio donde se utilizó la población finita, población tomada de los visitantes nacionales y extranjeros. El procedimiento fue mediante muestreo aleatorio simple, con un margen de error de $+/-5 \%$, un nivel de confianza del $95 \%$ y una varianza del $50 \%$. En este estudio una vez recogido los datos en la actividad de campo, fueron organizados, tabulados y analizados usando el programa SPSS Versión 18. Además, el tratamiento de los datos se realizó a través de la utilización de herramientas estadísticas univariantes y bivariantes.

\section{Resultados}

La gastronomía esmeraldeña es una de las más apetecidas por turistas nacionales y extranjeros lo cual ha motivado a la realización de la presente investigación desarrollada en los cantones Esmeraldas, Atacames y Rio Verde en Ecuador. Se realizó in situ y se obtuvieron resultados aproximados. Los resultados de las encuestas determinan que los tres cantones antes mencionados cuentan con potencial turístico y se puede fomentar la gastronomía. En cuanto a infraestructura turística la mitad de los encuestados la califican como regular, lo cual demuestra que es indispensable trabajar en este aspecto.

Los hallazgos revelan que los principales atractivos turísticos de la provincia de Esmeraldas son sus playas y la gastronomía, lo que permite combinar el turismo de sol y playa y turismo gastronómico. Al momento de calificar la viabilidad de la provincia de Esmeraldas la mayoría menciono que es regular mientras que en menor porcentaje manifestaron que es buena y muy buena; denotando prioridad este aspecto para que se pueda realizar el recorrido de la ruta gastronómica. Al ser consultados acerca del transporte adecuado para los turistas que la visitan desde diversos cantones de la provincia de Esmeraldas, respondieron que no existe un adecuado transporte, lo cual determina una imperiosa gestión para que los transportistas mejoren las unidades y amplíen turnos de recorridos.

A pesar de contar con algunas cadenas hoteleras de prestigio ubicadas en Atacames, Esmeraldas, Mompiche, al momento de analizar la calidad del servicio de alojamiento sostienen que es regular, bueno y muy pocos mencionan que es malo. Por otro lado, se consulto acerca de la existencia de alguna ruta gastronómica en la provincia de Esmeraldas para lo cual en su gran mayoría indicaron que no. Esto confirma que además de la provincia de Esmeraldas, aun no se planifica ni se diseña una ruta gastronómica en el Ecuador; en tal virtud se obtuvo un grado de aceptación alto en cuanto a la creación de una ruta gastronómica considerando que permitirá potenciar turísticamente a toda la provincia de Esmeraldas; también aseguraron que estarían interesados en recorrerla. Finalmente, los encuestados consideran que la creación de la ruta gastronómica permitirá dinamizar la economía local y generar empleo; esto conllevará a erradicar la pobreza y mejorar la calidad de vida de sus habitantes 


\section{Conclusiones}

Las rutas gastronómicas dinamizan economías y posicionan turísticamente los destinos; deben estar bien diseñadas, con una temática definida, señalización y señalética turística que permita disfrutar de paisajes naturales para valorizar la identidad local. Los actores deben colaborar y definir responsabilidades que se complementen con el apoyo del sector público y privado; todos estos factores son determinantes en el desarrollo sostenible y sustentable del producto turístico. La ruta gastronómica debe ofrecer actividades relacionadas a la degustación de platos típicos de la región, acompañadas de entretenimientos culturales y su entorno natural. En efecto Shenoy (2005), Kivela \& Crotts (2006) y Ulusoy (2008) aseveran que el turismo gastronómico puede convertirse en un tipo de turismo alternativo por sí solo para los destinos de sol y mar, y también puede servir como una actividad de apoyo a estos destinos.

La creación de una ruta gastronómica integrada por cantones Rio Verde, Esmeraldas y Atacames puede ubicar como destino turístico gastronómico a la Provincia de Esmeraldas por la potencialidad de los recursos turísticos, culturales naturales y exquisita gastronomía con la que cuenta, además los turistas podrán visitar otras localidades lo cual permitirá tener beneficiarios indirectos, considerando que los turistas son un elemento esencial en las rutas gastronómicas debido a que el principal gasto se enfoca en la alimentación. Es importante fomentar la inversión pública y privada que brinden facilidades turísticas para ofrecer un buen servicio y satisfacer necesidades de los visitantes.

En conclusión, las rutas gastronómicas contribuyen al desarrollo local debido a la diversidad de su oferta gastronómica (Jeambey, 2016). Es importante considerar la oportunidad que tiene el turista de ser el actor principal en vivir la experiencia de recorrer las rutas gastronómicas. Se propone como futuras líneas de investigación integrar la gastronomía con la cultura de cada región y de medir el grado de aceptación de las rutas que contribuya a fortalecer el producto turístico, también se debe trabajar en el inventario gastronómico por regiones para reconocer la potencialidad con la que se cuenta para la creación de futuras rutas gastronómicas.

\section{Referencias bibliográficas}

Akgol, Y., (2012). Gastronomi Turizmi ve Turkiye'yi Ziyaret Eden Yabancı Turistlerin Gastronomi Deneyimlerinin Degerlendirilmesi, Mersin University, Institute of Social Sciences, Department of Tourism and Hotel Management, MA Thesis, Mersin.

Akgoz, E., (2003). Turizm İsletmelerindeki Turk Gecelerinin Turk Kulturunu Tanıtmaya Katkıları, Selcuk University, Institute of Social Sciences, Department of Public Relations and Publicity, MA Thesis, Konya.

Ascanio, A. (2009). Rutas gastronómicas chilenas: una aproximación al tema. Pasos. Revista de turismo y patrimonio cultural, 7(2). 
Bernard, A., \& Domínguez, P. (2001). Rutas de arte y gastronomía Una propuesta inexplorada. El Turismo Rural en América Latina, 44.

Buiatti, S. (2011). Food and tourism: the role of the "Slow Food" association. In Food, Agri-Culture and Tourism (pp. 92-101). Springer, Berlin, Heidelberg.

Cagli, I.B., (2012). Turkiye'de Yerel Kulturun Turizm Odaklı Kalkınmadaki Rolu: Gastronomi Turizmi Ornegi, İstanbul Technical University, Institute of Science and Technology, Department of Urban and Region Planning, Region Planning, MA Thesis, İstanbul.

Cristóvão A., Tibério L., Abreu S. (2008) Restauração, Turismo e Valorização de Produtos Agro-alimentares Locais: o Caso do Espaço Transfronteiriço do DouroDuero. Pasos. Journal of Tourism and Cultural Heritage, 6(2).

Da Silva Oliveira, M., do Amaral Moretti, S. L., \& Pozo, H. (2015). A Hospitalidade e a Gestão de Restaurantes: Evidências de um Estudo Múltiplo de Caso em São Paulo, SP, Brasil. Rosa dos Ventos, 7(1).

Dos Santos, R. I. C., \& Antonini, B. O. (2004). La gastronomía típica de la Isla de Santa Catarina (Brasil). Estudios y Perspectivas en Turismo, 13, 89-110.

Folgado, J. A., Hernández, J. M., \& Campón, A. M. (2011). El turismo gastronómico como atractivo innovador del destino: un estudio empírico sobre rutas gastronómicas españolas. Comunicazione presentata al XIII seminario lusoespanhol de economía empresarial, tenutosi presso l'universidad de Évora, 2425.

Hall, C. M., Cambourne, B., Sharples, L., Macionis, N., \& Mitchell, R. (2003). Food Tourism Around the World development, management and markets.

Hernández Ramírez, J. (2011). Los caminos del patrimonio. Rutas turísticas e itinerarios culturales. Pasos. Revista de turismo y patrimonio cultural, 9(2).

Herrera, C., \& Arilla, J. M. P. (2013). La generación de lealtad a un destino de turismo gastronómico como factor clave en el desarrollo rural. Cuadernos aragoneses de economía, 23(1), 47-73.

Jeambey, Z. (2016). Rutas Gastronómicas y Desarrollo local: un ensayo de conceptualización en Cataluña. Pasos, 14(5), 1187.

Kim H, (1998). Perceived attractiveneses of Korean destinations. Annals of Tourism Research, 25 (2), pp. 340-361

Kivela, J., Crotts, J., (2006). Tourism and Gastronomy: Gastronomy's Influence on How Tourists Experience a Destination. Journal of Hospitality \& Tourism Research, 30: $354-377$.

Kozak, N., (1997). Genel Turizm İlkeler-Kavramlar, Turan Bookstore, Ankara

Krešić, D., \& Prebežac, D. (2011). Index of destination attractiveness as a tool for destination attractiveness assessment. Turizam: znanstveno-stručni časopis, 59(4), 497- 517. 


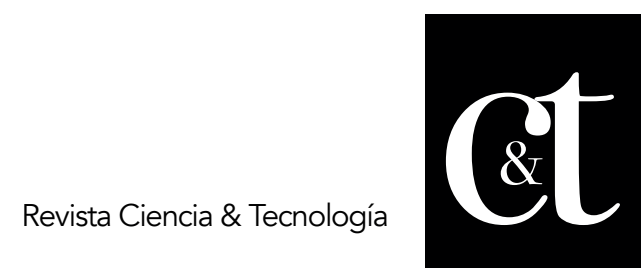

No. 21, 31 de enero de 2019

ISSN impreso: 1390 - 6321

López-Guzmán, T., \& Jesús, M. M. (2011). Turismo, cultura y gastronomía. Una aproximación a las rutas culinarias. Tourism \& Management Studies, 1.

Martins, U. M. O., Baptista, T., \& Rocha, M. M. (2011). La herencia de la gastronomía portuguesa en Brasil como un producto del turismo cultural. Estudios $y$ perspectivas en turismo, 20(2), 404-424.

Mascarenhas Tramontin, R. G., \& Gândara Gonçalves, J. M. (2010). Producción y transformación territorial: La gastronomía como atractivo turístico. Estudios y perspectivas en turismo, 19(5), 776-791.

Millán Vázquez de la Torre, M., Amador, L., \& Arjona Fuentes, J. M. (2016). La denominación de origen protegida "Los Pedroches" como ruta gastronómica del jamón ibérico: análisis del perfil del visitante y evolución futura. Cuadernos de Desarrollo Rural, 13(77).

Mitchell, M., \& Hall, D. (2005). Rural tourism as sustainable business: key themes and issues. Rural tourism and sustainable business, 3-14.

Schlüter, R. G., \& Thiel Ellul, D. (2008). Gastronomía y turismo en Argentina polo gastronómico Tomas Jofré. PASOS. Revista de Turismo y Patrimonio Cultural, 6(2es).

Shenoy, S., (2005). Food Tourism and The Culinary Tourist, a Thesis Presented to the Graduate School of Clemson University, USA.

Ulusoy, B., (2008). Ulke mutfagı tarih ve kulturu ortaya cıkartır, Food in life, Tourism gastronomy and life magazine, $\mathrm{V}: 3$, İstanbul.

World Tourism Organization (2013), UNWTO Annual Report 2012, UNWTO, Madrid.

Yuncu, H.R., (2010). Surdurulebilir turizm acısından gastronomi turizmi ve Persembe yaylası, Congress of Aybastı-Kabatas 11, Ankara Ministerio de Turismo. (2007). PLANDETUR 2020. Ecuador. 